\title{
USO DE FONTES RENOVÁVEIS DE CARBONO PROVENIENTES DE RESÍDUOS DAS INDÚSTRIAS DE ALIMENTOS E ÓLEO DE SOJA VISANDO À PRODUÇÃO DE POLI (3-HIDROXIBUTIRATO)
}

Manoela Estefânea Boff Zortéa ${ }^{1}$

Tárcio Enrico Savoldi ${ }^{2}$

Maryana Stella Gongoleski

Salah Din Mahmud Hasan ${ }^{2}$

Mônica Lady Fiorese ${ }^{2}$

\begin{abstract}
Resumo: Este trabalho teve como objetivo utilizar fontes renováveis de carbono para crescimento de Cupriavidus necator visando à produção de poli (3-hidroxibutirato) com menor custo, utilizando como suplementos resíduos provenientes das indústrias processadoras de alimentos tais como bagaço de mandioca e soro de queijo e uma matéria-prima também renovável, o óleo de soja. Os cultivos foram realizados seguindo um planejamento experimental $2^{3}$, visando obter nesta etapa a melhor condição de crescimento com os suplementos testados. Os resultados obtidos através do planejamento experimental mostraram que náo houve resultados significativamente diferentes, indicando que a utilização de qualquer um destes três suplementos (hidrolisado de bagaço de mandioca, soro de queijo e óleo de soja) náo influencia a resposta.
\end{abstract}

Palavras-chave: Poli (3-hidroxibutirato), Cupriavidus necator, suplementação.

Abstract: This study aimed to use alternative carbon sources for Cupriavidus necator growth aimed at producing poly (3-hydroxybutyrate) at a lower cost, using supplements like waste from food processing industries such as cassava bagasse and cheese whey and renewable raw material, the soybean oil. The cultures were carried out using an experimental design $2^{3}$, to obtain the best condition at this stage of growth with the supplements tested. The results obtained from the experimental design showed no significant results, indicating that the use of any of these three supplements (cassava bagasse hydrolysates, milk whey, and soy oil) did not influence the response.

Keywords: Poly (3-hydroxybutyrate), Cupriavidus necator, supplementation.

1 Universidade Federal do Paraná - UFPR / Programa de Pós-Graduaçấo em Engenharia de Alimentos; Centro Politécnico - Setor de Tecnologia - Bloco da Engenharia Química; Rua Francisco H. dos Santos - S/No. Jardim das Américas; CEP: 81531-980 - Curitiba - PR. manozortea@yahoo.com

2 Universidade Estadual do Oeste do Paraná - UNIOESTE / Centro de Engenharia e Ciências Exatas; Rua da Faculdade, 645; CEP: 85903-000 - Toledo PR.mlfiorese@gmail.com 


\section{Introduçáo}

Há muito tempo os materiais plásticos se tornaram parte importante em nosso dia-a-dia, pelas suas características de versatilidade, de resistência mecânica, durabilidade e resistência à degradação. No entanto, estas propriedades consideradas desejáveis agora são consideradas preocupantes, e o acúmulo de plásticos no ambiente tornou-se um problema mundial (Khanna \& Srivastava, 2005).

Em resposta à crescente preocupação com os problemas ambientais causados pela produção e acúmulo de materiais plásticos de origem petroquímica, bem como o esgotamento das fontes de combustíveis fósseis, muitos países estão realizando estudos de gerenciamento e diminuiçáo do volume de lixo sólido, buscando ainda a produção de materiais plásticos biodegradáveis, a partir de fontes renováveis de carbono. Portanto, estes novos materiais devem possuir as propriedades desejáveis dos plásticos convencionais, serem produzidos a partir de substratos renováveis e ainda serem completa e rapidamente biodegradados, quando descartados no meio ambiente (Piemolini, 2004).

Os plásticos biodegradáveis, ou bioplásticos, são polímeros que se degradam completamente ao ataque microbiano em um curto espaço de tempo, sob condiçôes apropriadas do meio ambiente, sua degradação é realizada principalmente pela atividade enzimática dos micro-organismos num período entre doze a dezoito meses (Lafferty et al., 1988; Byrom, 1990; Krupp \& Jeweell, 1992 citados por Fiorese, 2008).

Dentre os biopolímeros em desenvolvimento, estáo os polihidroxialcanoatos (PHAs), os quais são acumulados intracelularmente por bactérias produtoras, como reserva de carbono e/ou energia, na maioria das vezes, sob limitação de um nutriente essencial ao seu crescimento, como nitrogênio, fósforo, enxofre ou oxigênio (Lee et al., 1999).

O poli (3-hidroxibutirato) $(\mathrm{P}(3 \mathrm{HB}))$ constituído por monômeros de 3-hidroxibutirato, é o PHA mais bem caracterizado e o acumulado com maior freqüência por bactérias (Madigan et al., 2000). O Cupriavidus necator é um dos micro-organismos mais estudados para a produção de PHAs por apresentar alta velocidade de conversáo do substrato em células, utilizando normalmente fontes energéticas sintéticas (glicose/frutose) podendo utilizar fontes renováveis de carbono, que podem acumular cerca de $80 \%$ de sua massa seca em polímero (Ienczak, 2011).

Uma avaliação econômica do processo de produção de $\mathrm{P}(3 \mathrm{HB})$ indica que o custo do substrato tem uma contribuição importante no custo global da produção, podendo representar mais de $38 \%$, segundo Choi \& Lee (1999).

O alto custo de produçáo de PHAs pode ser minimizado usando resíduos orgânicos de baixo custo provenientes de indústrias processadoras de alimentos.

A mandioca (Manihot esculenta Crantz), também conhecida por aipim e macaxeira é uma cultura amplamente difundida por todo território nacional. Abrahão (2005) evidencia que uma fecularia que processa, em média, 200 toneladas de mandioca por dia, gera, aproximadamente, 183 toneladas por dia de bagaço, com $15 \%$ de matéria seca; $73 \%$ de amido; e, $15 \%$ de fibras na matéria seca.

De acordo com dados divulgados pela Embrapa Gado de Leite, em 2011 no Brasil foram produzidos aproximadamente 675 milhóes de quilos de queijo (CONAB, 2012). Partindo do princípio que são necessários 10 litros de leite para produzir 1 quilo de queijo, e que o restante é soro, a produçáo de soro de leite em 2011 foi de aproximadamente 6.075 milhôes de litros (BRASIL, 2010).

A soja é um grão muito versátil que dá origem a produtos e subprodutos muito usados pela agroindústria, indústria química e de alimentos. Recentemente, a soja vem crescendo também como fonte alternativa de combustível. Segundo ABIOVE (2012) na atualidade existe um excedente de 232 mil toneladas de óleo de soja no Brasil.

Esta pesquisa teve por objetivo utilizar fontes renováveis de carbono para crescimento de Cupriavidus necator visando à produção de poli (3-hidroxibutirato) com e sem suplementação do meio, utilizando resíduos das indústrias processadoras de alimentos, tais como: bagaço de mandioca hidrolisado e soro de queijo, além de suplementação com óleo de soja, a fim de estudar as condiçôes de crescimento do micro-organismo em meio alternativo ao sintético (glicose/frutose) o que pode contribuir no decréscimo do custo da produção de plástico biodegradável em meio suplementado com substratos de baixo custo, bem como fornecer uma opçáo interessante de aumento de valor agregado às indústrias geradoras dos resíduos estudados nesta pesquisa.

\section{Materiais e métodos}

Os resíduos, soro de queijo e bagaço de mandioca, foram cedidos pelas empresas Laticínio Lacto Mesa e Fecularia Zadimel de Toledo/PR, respectivamente. 


\subsection{Hidrólise do bagaço de mandioca}

A hidrólise do bagaço de mandioca foi realizada de acordo com estudos realizados por Krueger (2009). Após, filtrou-se à vácuo para separação dos sólidos e deixou-se resfriar. A solução obtida foi neutralizada com adição de $\mathrm{NaOH} 10 \mathrm{~N}$. Este procedimento deu-se em função do micro-organismo utilizado neste estudo possuir capacidade de assimilar em seu metabolismo apenas fontes de carbono redutoras.

\subsection{Caracterizaçáo dos resíduos utilizados como suplementos}

A caracterização dos resíduos, soro de queijo e bagaço de mandioca hidrolisado (hidrolisado amiláceo) foi realizada através da mediçáo de $\mathrm{pH}$ (TECNOPON, modelo MPA-210), determinação da alcalinidade e acidez (IAL, 1985), sólidos totais e sólidos totais voláteis (APHA, 2005), açúcares redutores (Miller, 1959), demanda química de oxigênio - DQO, demanda bioquímica de oxigênio - DBO (APHA, 2005), nitrogênio e fosfato total (Mackereth et al., 1978).

\subsection{Micro-organismo e meios de cultura}

A cepa utilizada foi Cupriavidus necator DSM 545, que foi doada pelo Laboratório de Engenharia Bioquímica (ENGEBIO) da Universidade Federal de Santa Catarina (UFSC).

Os experimentos foram realizados utilizando dois pré-cultivos. No primeiro, as células foram inoculadas em caldo nutriente (NB), contendo peptona de carne $\left(5,0\right.$ g.L $\left.\mathrm{L}^{-1}\right)$ e extrato de carne $(3,0$ g. $\left.L^{-1}\right)$ e, como segundo pré-cultivo, foi utilizado o meio mineral (MM) adicionado dos nutrientes que o compóem (em g. $\left.\mathrm{L}^{-1}\right): \mathrm{KH}_{2} \mathrm{PO}_{4} 0$, 44; citrato de amônio e ferro III 0,06; cloreto de cálcio 0,01 ; sulfato de magnésio 0,5 ; ureia 2,3 ; ácido nitrilotriacético 0,19 ; soluçáo de fonte de carbono 40,0; elementos traço $1 \mathrm{~mL} . \mathrm{L}^{-1}$ e água. A solução de elementos traço apresentou em sua composição (em g. $\left.\mathrm{L}^{-1}\right): \mathrm{H}_{3} \mathrm{BO}_{3} 0,3 ; \mathrm{CoCl}_{2} \cdot 6 \mathrm{H}_{2} \mathrm{O} 0,2 ; \mathrm{ZnSO}_{4} \cdot 7 \mathrm{H}_{2} \mathrm{O}$ 0,$1 ; \mathrm{MnCl}_{2} \cdot 4 \mathrm{H}_{2} \mathrm{O} 0,03 ; \mathrm{Na}_{2} \mathrm{MoO}_{4} \cdot 2 \mathrm{H}_{2} \mathrm{O} 0,03$; $\mathrm{NiCl}_{2} \cdot 6 \mathrm{H}_{2} \mathrm{O}$ 0,02; e CuSO $.5 \mathrm{H}_{2} \mathrm{O} 0,01$ (Aragão, 1996 adaptado por Ienczak, 2011).

Para a realizaçáo dos cultivos de crescimento, utilizou-se o mesmo meio descrito, porém com alteraçóes na adição cada um dos suplementos segundo planejamento experimental $2^{3}$, totalizando oito ensaios adicionados da triplicata no ponto central. Sendo assim, as quantidades de água e de solução da fonte de carbono foram ajustadas conforme cada ensaio programado, uma vez que, o teor de açúcar redutor do hidrolisado amiláceo e do soro de queijo foram considerados.

\subsection{Condiçóes dos cultivos}

Os pré-cultivos e cultivos foram conduzidos de acordo com o fluxograma apresentado na Figura 1.

Figura 1 - Fluxograma exemplificando as condições de cultivo do micro-organismo Cupriavidus necator visando à produção de $\mathrm{P}(3 \mathrm{HB})$.

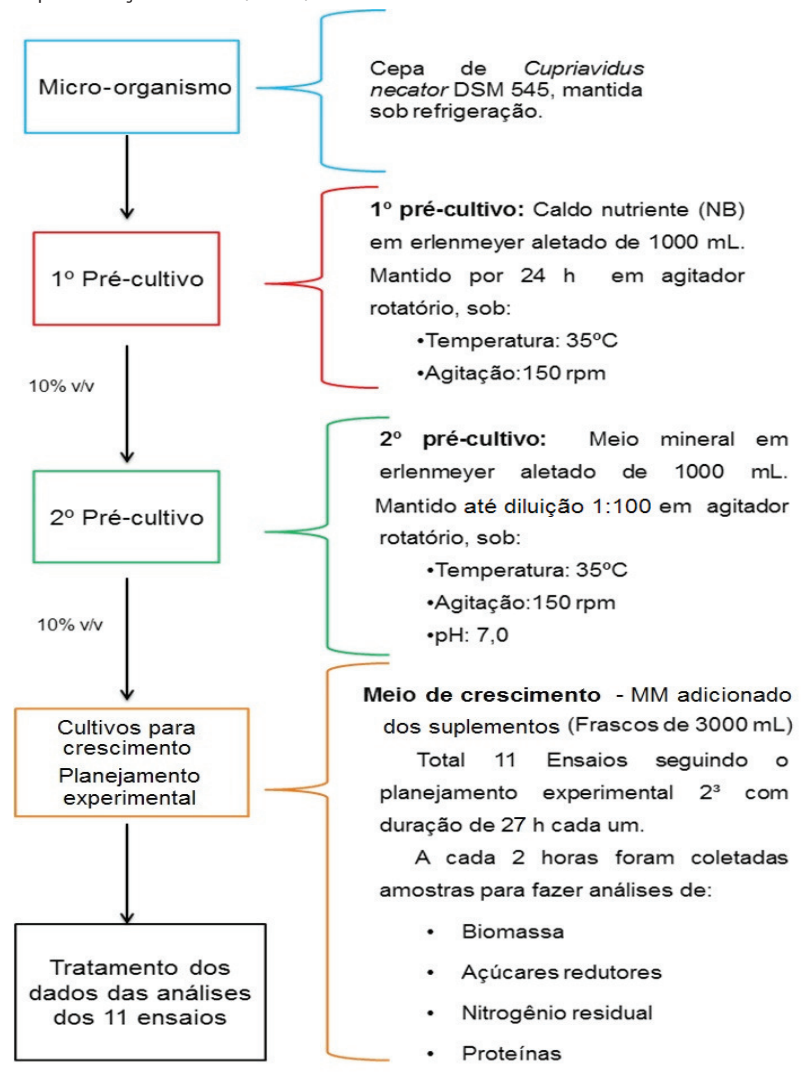

Os cultivos de crescimento foram realizados em frascos de vidro adaptados, de forma a obter-se um sistema de vedaçáo passível de troca de gases e com possibilidade de retirada de amostra ao longo do tempo. As condiçóes de cultivo foram as mesmas utilizadas nos pré-cultivos. $\mathrm{O} \mathrm{pH}$ foi mantido na faixa entre $6,0-7,0$ pela adição manual de $\mathrm{NaOH}$ $1,25 \mathrm{~N}$ ou $\mathrm{HCl} 2,7 \mathrm{~N}$.

\subsection{Planejamento experimental}

Um planejamento experimental $2^{3}$ com triplicata no ponto central foi aplicado para avaliar a influência do: hidrolisado amiláceo $(\mathrm{H})$, soro de queijo (S) e óleo de soja (OS), conforme mostra a Tabela 1 , em seus respectivos níveis codificados e reais. 
Tabela 1 - Níveis codificados reais das variáveis para planejamento experimental fatorial completo $2^{3}$

\begin{tabular}{lcc}
\hline \multicolumn{1}{c}{ FATOR } & Nível INFERIOR (-1) & Nível SUPERIOR (+1) \\
\hline Hidrol. Amiláceo (H) (\%) & 0 & 10 \\
Soro de queijo (S) (\%) & 0 & 10 \\
Óleo de soja (OS) (\%) & 0 & 10 \\
\hline
\end{tabular}

As análises estatísticas para todos os planejamentos propostos foram realizadas utilizando-se o software Statistica $8.0^{\oplus}$.

\subsection{Técnicas analíticas}

Para a determinação da biomassa foram utilizados um método indireto realizado pela leitura da absorbância das amostras em espectrofotômetro (modelo UV-1800, marca Shimadzu, Japão) a 600 $\mathrm{nm}$ e outro direto por gravimetria. Ambos realizados de acordo com as metodologias descritas por Squio, (2003). A determinação dos açúcares redutores totais foi realizada pelo método do ácido 3,5-dinitrossalicílico (DNS) (Miller, 1959). O nitrogênio determinado no meio de cultura é o nitrogênio amoniacal. A dosagem deste foi realizada utilizando-se o kit Ureia ES (Gold Analisa), que determina uréia pelo método enzimático-colorimétrico. A determinação da dosagem de proteínas foi realizada pelo método de Biureto (Stickland, 1951).

\section{Resultados e discussóes}

\subsection{Caracterizaçáo dos resíduos}

A caracterização dos resíduos em estudo é de fundamental importância, pois grande parte dos micro-organismos, dentre eles, Cupriavidus necator, necessitam de limitação de algum nutriente essencial assim como, excesso de carbono no meio em que se desenvolvem, para que então, haja regulaçáo de seu metabolismo e inicie o processo de absorção de carbono e acúmulo do mesmo em forma de grânulos como reserva de energia (Krueger, 2009). Na Tabela 2 encontra-se apresentado a caracterização dos resíduos em estudo.

Devido ao elevado teor de matéria orgânica constatado nos resíduos em estudo após a caracterização, é possível considerar que estes, tornam-se aplicáveis à produção biotecnológica de compostos de maior valor agregado, como é o caso do $\mathrm{P}(3 \mathrm{HB})$.

A análise da quantificação de açúcares redutores mostrou que tanto o hidrolisado amiláceo quanto o soro de queijo, apresentaram teores de açúcar consideráveis, 21,18 g.L $L^{-1}$ e 39,54 g.L $L^{-1}$, respectivamente, caracterizando-os como interessantes fontes de carbono para o crescimento do micro-organismo.

\subsection{Comportamento do Cupriavidus necator em diferentes composiçóes de cultivos}

$\mathrm{O}$ processo de produção de $\mathrm{P}(3 \mathrm{HB})$ por Cupriavidus necator é conduzido em duas fases distintas: fase de crescimento não limitado com produção de biomassa e fase de produção com acúmulo de polímero. Segundo Fiorese (2008), se a primeira fase tiver uma produtividade elevada de células haverá uma melhoria na produtividade global do polímero. Portanto, é interessante utilizar condiçóes de cultivo que proporcionem valores de velocidade de crescimento $\left(\mu_{\text {máx }}\right)$ altos, bem como quantidades expressivas de células nesta fase.

Tabela 2 - Caracterização dos resíduos utilizados como suplementos

\begin{tabular}{|c|c|c|}
\hline ANÁLISE & BAGAÇO DE MANDIOCA HIDROLISADO & SORO DE QUEIJO \\
\hline PH & - & 4,35 \\
\hline ALCALINIDADE & $37,5 \mathrm{mg} \mathrm{CaCO3}$ & $475,0 \mathrm{mg} \mathrm{CaCO3}$ \\
\hline ACIDEZ & $1550,0 \mathrm{mg} \mathrm{CH} 3 \mathrm{COOH}$ & $2710,0 \mathrm{mg} \mathrm{CH} 3 \mathrm{COOH}$ \\
\hline SÓLIDOS TOTAIS & 0,74 \% ou 7423 mg.L-1 & 6,71 \% ou 68280 mg.L-1 \\
\hline SÓLIDOS VOLÁTEIS & 0,67 \% ou 6710 mg.L-1 & $6,32 \%$ ou 64265 mg.L-1 \\
\hline AÇÚCAR REDUTOR & 2,1\% ou 21,176 g.L-1 & $3,9 \%$ ou 39,534 g.L-1 \\
\hline DQO & 7448,00 mg.L-1 de 02 & 82080,00 mg.L-1 de 02 \\
\hline DBO & $11320 \mathrm{mg.L}-1$ & 58734 mg.L-1 \\
\hline NITROGÊNIO TOTAL & $302,57 \mathrm{mg.L}-1$ & $935,20 \mathrm{mg} . \mathrm{L}-1$ \\
\hline FOSFATO TOTAL & $26,86 \mathrm{mg} . \mathrm{L}-1$ & $250,07 \mathrm{mq} . \mathrm{L}-1$ \\
\hline
\end{tabular}

Com o intuito de verificar a influência dos suplementos utilizados neste estudo e seu comportamento na fase de crescimento de Cupriavidus necator onze ensaios foram realizados, conforme planejamento experimental $2^{3}$.

Os resultados obtidos nos onze ensaios encon- tram-se apresentados na Figura 2 (A, B, C, D, E, F, G, H, I, J e K), onde estáo plotadas as curvas características de evolução das concentrações de biomassa total e proteína, e os consumos da concentração de substrato e nitrogênio ao longo do tempo nos onze cultivos; Os cultivos foram iniciados com aproximadamente 40 g. $\mathrm{L}^{-1}$ de fonte de carbono. 
Figura 2 - Evoluções da biomassa total (XT) e proteína; consumos das concentrações de nitrogênio e substrato, expressos em g.L.-1 em função do tempo. A - Ensaio 1 (sem suplemento); B - Ensaio 2 ( H 10\%); C - Ensaio 3 (S 10\%); D - Ensaio 4 (H 10\% + S 10\%); E - Ensaio 5 (OS 10\%); F - Ensaio 6 (H 10\%+ 10\% OS); G - Ensaio 7 (S 10\% + 10\% OS); H - Ensaio 8 (H 10\% + S 10\% + OS 10\%); I - Ensaio 9 (H 5\% + S 5\% + OS 5\%); J - Ensaio 10 (H 5\% + S 5\% + OS 5\%); K- Ensaio 11 (H 5\% + S 5\% + OS 5\%).
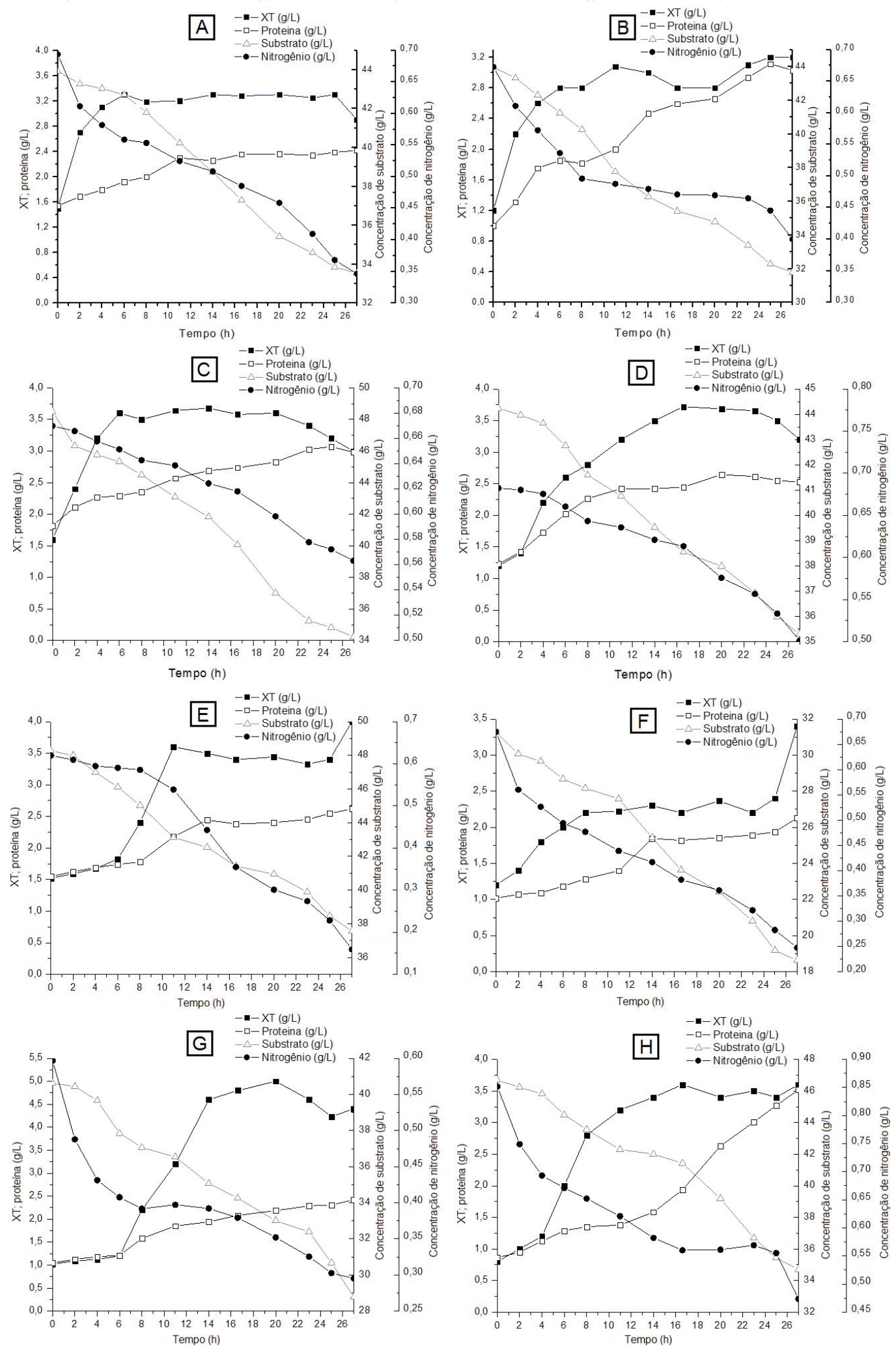

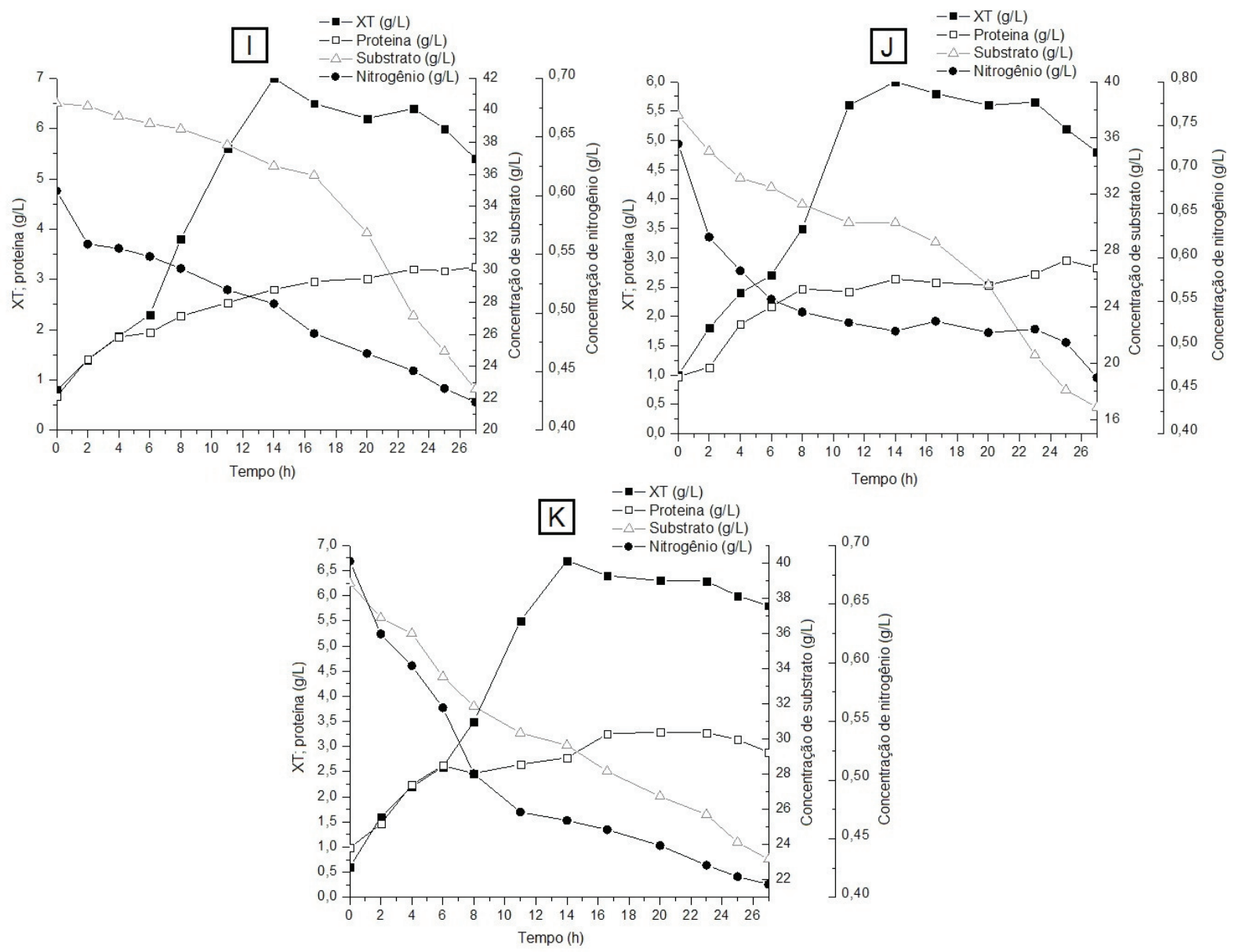

Observou-se através da Figura 2 (A, B, C, D, E, F, G, H, I, J e K), que em todos os onze ensaios compostos por diferentes meios de cultivo realizados para o crescimento de $C$. necator foi verificada uma curva característica de crescimento celular microbiano, na qual a biomassa total aumentou exponencialmente, havendo também um aumento do conteúdo protéico ao longo do tempo de experimento, sendo que o mesmo seguiu o comportamento da curva de biomassa total. Enquanto que as concentraçóes de substrato e nitrogênio foram diminuindo devido ao consumo para síntese celular. Cabe ressaltar que a curva de consumo de nitrogênio contém valores de concentração expressos de forma qualitativa, e não quantitativa, isto se dá, devido a análise ser realizada por kit enzimático-colorimétrico, sendo utilizada apenas como um indicativo de consumo e náo de exatidão, como já observado por Dalcanton (2006), Fiorese (2008), Ienczak (2011).

Os resultados da fase de crescimento obtidos neste planejamento experimental desenvolvido, com relação à duração das fases (lag, pré-exponencial e $\log$ ) e velocidade específica de crescimento encontram-se resumidamente apresentados na Tabela 3.

Tabela 3 - Resumo da fase de crescimento (fase lag, pré-exponencial, log e total de crescimento (lag+pré-exp+log)) dos 11 ensaios do planejamento experimental

\begin{tabular}{|c|c|c|c|c|c|c|}
\hline ENSAIO & COMPOSIÇÃO $(\mathrm{H}, \mathrm{S}, 0 \mathrm{OS})(\%)$ & DURAÇÃO FASE LAG (H) & DURAÇÄ0 FASE PRÉ-EXPONENCIAL (H) & DURAÇÃO FASE LOG (H) & TEMPO TOTAL DE CRESCIMENTO (H) & $M(H-1)$ \\
\hline 01 & $0,0,0$ & 0 & 0 & 6 & 6 & 0,18 \\
\hline 02 & $10,0,0$ & 0 & 0 & 6 & 6 & 0,19 \\
\hline 03 & $0,10,0$ & 0 & 0 & 6 & 6 & 0,17 \\
\hline 04 & $10,10,0$ & 0 & 2 & 4 & 6 & 0,22 \\
\hline 05 & $0,0,10$ & 6 & 0 & 5 & 11 & 0,21 \\
\hline 06 & $10,0,10$ & 0 & 0 & 8 & 8 & 0,14 \\
\hline 07 & $0,10,10$ & 6 & 0 & 8 & 14 & 0,16 \\
\hline 08 & $10,10,10$ & 0 & 4 & 7 & 11 & 0,21 \\
\hline 09 & $5,5,5$ & 0 & 0 & 14 & 14 & 0,14 \\
\hline 10 & $5,5,5$ & 0 & 0 & 11 & 11 & 0,14 \\
\hline 11 & $5,5,5$ & 0 & 0 & 14 & 14 & 0,13 \\
\hline
\end{tabular}


Os resultados apresentados na Tabela 3 mostram que o Cupriavidus necator pode crescer nos diferentes meios suplementados testados neste estudo, acredita-se porém, que devido ao fato dos cultivos terem composiçóes diferentes e por consequência o micro-organismo assimilar mais rapidamente ou lentamente os substratos que os compóem, alguns ensaios apresentaram fase lag e diferentes velocidades especificas máximas de crescimento.

Pode-se perceber que com exceção dos ensaios $06,09,10$ e 11 os demais ensaios apresentaram velocidades de crescimento coerentes com os valores encontrados por Oliveira (1999); Marangoni et al. (2002); Kim et al. (1994) e Schneider (2006), se não estão dentro, encontram-se próximas da faixa 0,19$0,23 \mathrm{~h}^{-1}$. Os ensaios que não se enquadraram dentro da referência, continham óleo em sua composição.
Devido a este ter densidade diferente e também ser insolúvel no meio e na água de lavagem das células para determinaçáo de biomassa seca, o mesmo pode não ter sido totalmente removido durante esta determinação analítica, ocasionando erro na sua quantificação; outra justificativa por fim, como também citado por Finkler (2002), cabe lembrar que o presente trabalho foi realizado em frascos náo aletados, o que pode ter causado a diminuiçáo da velocidade específica de crescimento do micro-organismo, uma vez que o óleo cria uma barreira, dificultando ainda mais a troca de gases dentro do frasco.

Os resultados obtidos através do planejamento experimental de biomassa total (XT) durante a fase de crescimento dos 11 ensaios (Tabela 4) foram analisados de forma estatística e estão apresentados a seguir.

Tabela 4 - Matriz de dados da fase de crescimento

\begin{tabular}{ccccc}
\hline ENSAIO & HIDROLISADO & SORO & 0́LEO & BIOMASSA TOTAL - XT (G.L-1) \\
\hline 01 & -1 & -1 & -1 & 3,3 \\
02 & 1 & -1 & -1 & 2,8 \\
03 & -1 & 1 & -1 & 3,6 \\
04 & 1 & 1 & -1 & 2,6 \\
05 & -1 & -1 & 1 & 3,6 \\
06 & 1 & -1 & 1 & 2,2 \\
07 & -1 & 1 & 1 & 4,6 \\
08 & 1 & 1 & 1 & 3,2 \\
09 & 0 & 0 & 0 & 7,0 \\
10 & 0 & 0 & 0 & 5,6 \\
11 & 0 & 0 & 0 & 6,7 \\
\hline
\end{tabular}

Fonseca (2003), em sua pesquisa utilizando de E. coli recombinante proveniente de inserção de plasmídeos de $C$. necator, em meio suplementado com amido hidrolisado, soro de queijo e óleo de soja em seus níveis máximos ( $5 \%, 5 \%$ e $5 \%$ ), obteve 4,63 g. $\mathrm{L}^{-1}$ como valor de biomassa, no presente estudo, percebeu-se que nas mesmas proporçôes dos mesmos três suplementos $(5 \%, 5 \%$ e $5 \%)$ utilizados nos cultivos (ensaios 09, 10 e 11), as maiores quantidades de biomassa produzidas (7,0 g. $\mathrm{L}^{-1}, 5,6$ g.L $\mathrm{L}^{-1}$ e 6,7 g. $\mathrm{L}^{-1}$, respectivamente) também foram conseguidas e de acordo com Marangoni et al. (2000) e Kahar et al. (2004), a utilizaçáo de suplementos pode interferir na via metabólica proporcionando maior produção de células e resultando em maior produtividade.

A Tabela 5 mostra os efeitos obtidos através da análise estatística para cada uma das respostas observadas quanto à biomassa total produzida durante a fase de crescimento, enquanto que a Figura 3 ilustra esta análise através do Gráfico de Pareto.

Tabela 5 - Efeitos e coeficientes $(a=0,10)$

\begin{tabular}{|c|c|c|c|c|c|c|}
\hline & EFEITO & $\begin{array}{c}\text { ERRO PADRÃO } \\
\text { DO EFEITO }\end{array}$ & $T(3)$ & $\mathbf{P}$ & COEF. & ERRO PADRÃO DO COEF. \\
\hline Média/Interc. & 4,10909 & 0,841542 & 4,882814 & 0,016424 & 4,109091 & 0,841542 \\
\hline (1)HIDROL & $-1,07500$ & 1,973590 & $-0,544693$ & 0,623845 & $-0,537500$ & 0,986795 \\
\hline (2)SORO & 0,52500 & 1,973590 & 0,266013 & 0,807464 & 0,262500 & 0,986795 \\
\hline (3)ÓLEO & 0,32500 & 1,973590 & 0,164675 & 0,879670 & 0,162500 & 0,986795 \\
\hline 1 by 2 & $-0,12500$ & 1,973590 & $-0,063336$ & 0,953483 & $-0,062500$ & 0,986795 \\
\hline 1 by 3 & $-0,32500$ & 1,973590 & $-0,164675$ & 0,879670 & $-0,162500$ & 0,986795 \\
\hline 2 by 3 & 0,47500 & 1,973590 & 0,240678 & 0,825315 & 0,237500 & 0,986795 \\
\hline $1^{*} 2^{*} 3$ & 0,12500 & 1,973590 & 0,063336 & 0,953483 & 0,062500 & 0,986795 \\
\hline
\end{tabular}

$\mathrm{R}^{2}=0,139$ 
Figura 3 - Gráfico de Pareto mostrando os efeitos sobre as fases de crescimento

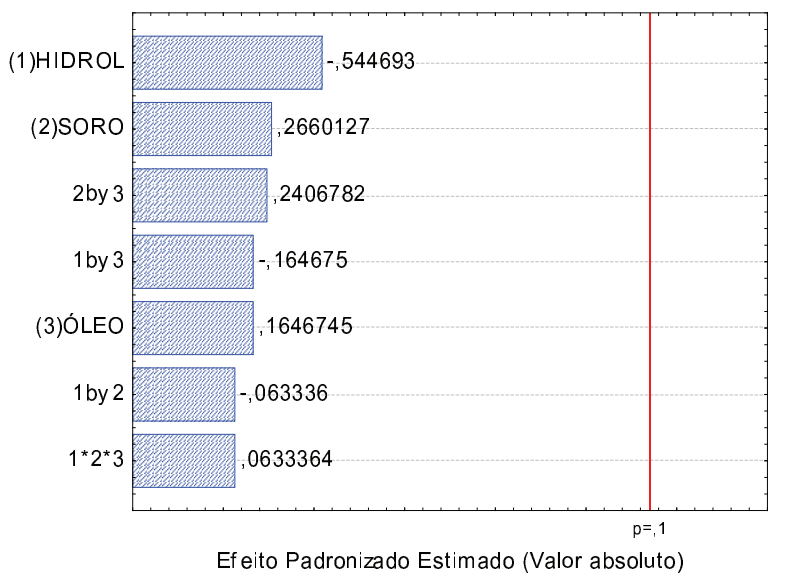

De acordo com a análise estatística apresentada na Tabela 5 e na Figura 3, na faixa estudada, nos extremos $-1 \mathrm{e}+1$, não há resultados significativos, indicando que nestes dois níveis, todas as três variáveis (fatores - hidrolisado, soro de queijo e óleo de soja) náo influenciam a resposta. No entanto, a presença do ponto central permite o cálculo da curvatura.

A Tabela 6 mostra os efeitos obtidos através da análise estatística incluindo a curvatura, enquanto que a Figura 4 ilustra esta análise através do Gráfico de Pareto.

Tabela 6 - Efeitos e coeficientes $(a=0,10)$

\begin{tabular}{ccccccc}
\hline & EFEITO & ERRO PADRÃ0 DO EFEITO & $\mathrm{T}(3)$ & $\mathbf{P}$ & COEF. & ERRO PADRÃo DO COEF. \\
\hline Média/Interc. & 3,23750 & 0,260608 & 12,42286 & 0,006417 & 3,237500 & 0,260608 \\
Curvatura & 6,39167 & 0,998054 & 6,40413 & 0,023526 & 3,195833 & 0,499027 \\
(1)HIDROL & $-1,07500$ & 0,521217 & $-2,06248$ & 0,175260 & $-0,537500$ & 0,260608 \\
(2)SORO & 0,52500 & 0,521217 & 1,00726 & 0,419866 & 0,262500 & 0,260608 \\
(3)ÓLE0 & 0,32500 & 0,521217 & 0,62354 & 0,596564 & 0,162500 & 0,260608 \\
1 by 2 & $-0,12500$ & 0,521217 & $-0,23982$ & 0,832806 & $-0,062500$ & 0,260608 \\
1 by 3 & $-0,32500$ & 0,521217 & $-0,62354$ & 0,596564 & $-0,162500$ & 0,260608 \\
2by3 & 0,47500 & 0,521217 & 0,91133 & 0,458321 & 0,237500 & 0,260608 \\
$1 * 2 * 3$ & 0,12500 & 0,521217 & 0,23982 & 0,832806 & 0,062500 & 0,260608 \\
\hline
\end{tabular}

$$
\mathrm{R}^{2}=0,96
$$

Figura 4 - Gráfico de Pareto mostrando os efeitos sobre as fases de crescimento, incluindo a curvatura

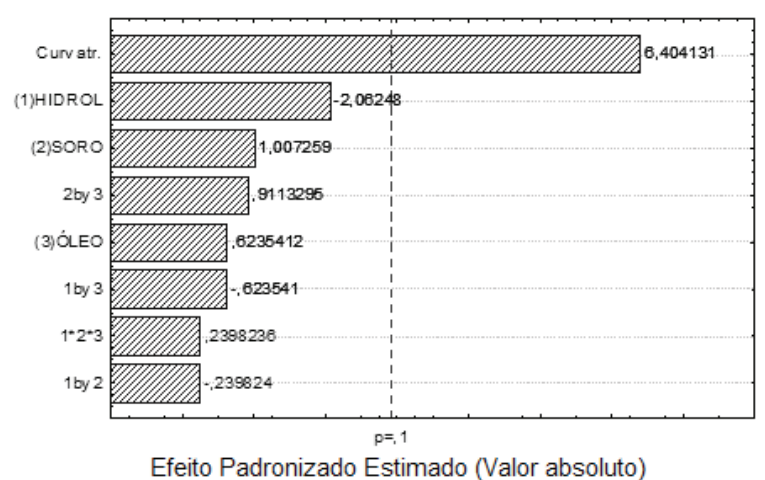

O tipo de planejamento usado, com níveis -1 $\mathrm{e}+1$, geram modelos lineares. A triplicata no ponto central (inserção de um terceiro nível) permite calcular a curvatura, ou seja, quando o modelo linear náo ajusta os pontos experimentais a curvatura se mostra significativa (com baixo p-valor), indicando que um modelo não linear (quadrático) ajustaria melhor os pontos experimentais.

\section{Conclusóes}

A caracterização dos resíduos apontou a viabilidade dos mesmos serem utilizados como nutrientes pelo micro-organismo para seu crescimento celular; na faixa estudada do planejamento experimental, não houve resultados significativos, indicando que a utilização de quaisquer destes três suplementos (hidrolisado, soro de queijo e óleo de soja) não influencia a resposta; o melhor resultado para produçáo de biomassa foi obtido no experimento onde as concentraçóes de suplementos encontravam-se em seus níveis centrais $(5 \% \mathrm{H}+$ $5 \% \mathrm{~S}+5 \%$ OS); o cálculo da curvatura indica que um modelo não linear (quadrático) ajustaria melhor os pontos experimentais.

Como conclusão final deste trabalho, tem-se que a utilização de resíduos das indústrias de alimentos é uma alternativa viável para o crescimento de $C$. necator e, por conseguinte, produção de poli (3-hidroxibutirato), conseguindo-se assim, redução nos custos do processo a fim de torná-los mais competitivos no mercado, além do beneficio de redução no tratamento de efluentes destes resíduos e da valorização dos mesmos. A utilizaçáo de óleo de soja, apesar de não ser um resíduo, é extremamente promissora, pois trata-se também de uma fonte renovável abundante no Brasil. 


\section{Referências}

ABIOVE. Associação Brasileira das Indústrias de Óleos Vegetais. Disponível em: <http://www.abiove. com. br/menu_br.html $>$. Acesso em: 21 nov. 2012.

Abrahão, J. J. dos S. Bagaço da mandioca gerado em fecularias alimenta bovinos de corte. Revista ABAM, ano III, no 11, 2005. Disponível em: <http:// www.abam.com.br/ revista/revista11/iapar.php>. Acesso em: 22 nov. 2012.

APHA (American Public Health Association); AWWA (American Water Works Association); WEF (Water Environment Federation). Standard Methods for the Examination of Water and Wastewater. 21 ${ }^{\mathrm{a}}$ Ed. Washington - DC: APHA, 2005.

Aragão, G. M. F. Production de polyhydroxyalcanoates par Alcaligenes eutrophus: caractérisation cinétique et contribution à l'optimisation de la mise en oeuvre des cultures. Toulouse: L'institut National des Sciences appliquees de Toulouse. Tese (Doutorado), 1996.

BRASIL, Ministério da Agricultura, Pecuária e Abastecimento. Produção brasileira de queijos. Disponível em: http://www.cnpgl.embrapa.br. Acesso em: 22 nov. 2012.

Choi, J.; Lee, S. Y. Factors affecting the economics of polyhydroxyalkanoate production by bacterial fermentation. Applied Microbiology and Biotechnology, v. 51, p. 13-21, 1999.

CONAB, Companhia Nacional de Abastecimento. Estudo de Prospecção de Mercado, Safra 2012/2013. Disponível em: <http://www.conab. gov.br>. Acesso em 22 nov. 2012.

Dalcanton, F. Produção, extração e caracterização de poli (3-hidroxibutirato) por Ralstonia eutropha em diferentes substratos. Florianópolis, 2006. 132 p. Dissertação (Mestrado), UFSC.

Finkler, L. Utilização do potencial de óxido-redução para o monitoramento de culturas de Ralstonia eutropha visando a produçáo de polihidroxibutirato. Florianópolis, 2002.78 p. Dissertação (Mestrado), UFSC.

Fiorese, M. L. Estratégias de cultivo e recuperação e caracterização de poli (3-hidroxibutirato) produzido por Ralstonia eutropha. Florianópolis, 2008. p. 180. Tese (Doutorado), UFSC.

Fonseca, G. G. Produção de polihidroxialcanoatos por Escherichia coli recombinante. Florianópolis, 2003. 154 p. Dissertação (Mestrado), UFSC.

Ienczak, J. L. Estratégia de cultivo em alta concentração celular para a produção de Poli (3-hidroxibutirato) em substratos com moderada concentração da fonte de carbono. Florianópolis, 2011. 71 p. Tese (Doutorado), UFSC.
Instituto Adolfo Lutz - IAL. Normas analíticas do Instituto Adolfo Lutz. São Paulo: IMESP, $3^{a}$ ed., v. 1: Métodos químicos e físicos para análise de alimentos. 1985. p. 533.

Kahar, P.; Tsuge, T.; Taguchi, K.; Doi, Y. High yield production of polyhydroxyalkanoates from soybean oil by Ralstonia eutropha and its recombinant strain. Polymer Degradation and Stability, v. 83. P. 79-86. 2004.

Khanna, S.; Srivastava, A. K. Computer simulated fed-batch cultivation for over production of PHB: a comparison of simultaneous and alternate feeding of carbon and nitrogen. Biochemical Engineering Journal, v. 27, p.197-203, 2005.

Kim, B. S.; Lee, S. C; Lee, S. Y.; Chang, H. N.; Chang, Y. K.; Woo S. I. Production of polyhydroxybutyrate by fed batch with glucose concentration control in Ralstonia eutropha. Biotechnology Bioeng, v. 43, p. 892-898. 1994.

Krueger, C. L. Seleção de linhagens de bacillus produtoras de polihidroxi-alcanoatos a partir de resíduo do processamento de mandioca. Florianópolis, 2009. 98 p. Dissertação (Mestrado), UFSC.

Lee, S. Y.; Choi, J.; Wong, H. H. Recent advances in polyhydroxyalkanoate production by bacterial fermentation: minireview. International Journal of Biological Macromolecules, n. 25, p. 31-36, 1999.

Mackereth, F. J. H.; Heron, J.; Talling, J. F. Water analysis: some revised methods for limnologists. Scientific Publication, v. 36.Cumbria, 1978. 120 p.

Madigan, M. T.; Martinko, J. M.; Parker, J. B. Biology of Microorganisms, Prentice-Hall International Editions, Upper Saddle River, 2000.

Marangoni, C.; Furigo, Jr, A.; Aragão, G. M. F. Oleic acid improves poly(3-hydroxybutyrate-co-3-hydroxyvalerate) production by Ralstonia eutropha in inverted sugar and propionic acid. Biotechnology Letters, v. 22, p. 1635-1638. 2000.

Marangoni, C.; Furigo, Jr, A.; Aragão, G. M. F. Production of poly (3-hydroxybutyrate-co-3-hydroxyvalerate) by Ralstonia eutropha in whey and inverted sugar with propionic acid feeding. Process Biochemistry, v. 38, p. 137-141. 2002.

Miller, G. L. Analytical Chemistry. v. 31, p. 426428, 1959.

Oliveira, R. Produção de poli-3-hidroxibutirato $(\mathrm{P}(3 \mathrm{HB}))$ por Ralstonia eutropha em substrato de baixo custo: Estudos de diferentes estratégias de limitação de crescimento. Florianópolis, 1999. 100 p. Dissertação (Mestrado), UFSC. 
Piemolini, L. T. Modelagem estrutural da PHA sintase de Choromobacterium violaceun para estudos de mutação sítio-dirigida. Florianópolis, 2004. Dissertação (Mestrado), UFSC.

Schneider, A. L. S. Síntese, caracterização e biodegradação de poli-3-hidroxibutirato obtido por Ralstonia eutropha. Florianópolis, 2006. 220 p. Tese (Doutorado), UFSC.
Squio, C. R. Estratégias de alimentação e utilização de suplementos nutricionais na produção de poli $(3-\mathrm{Hi}-$ droxibutirato-co-3-Hidroxivalerato) por Ralstonia eutropha. Florianópolis, 2003. 161 p. Dissertação (Mestrado), UFSC.

Stickland, L. H. The determination of small quantities of bactéria by means of biuret reaction. J. Gen. Microbiol. v. 5, p. 698-703. 1951. 